UUITP-07/02

hep-th/0205227

\title{
Inflation, holography, and the choice of vacuum in de Sitter space
}

\author{
Ulf H. Danielsson \\ Institutionen för teoretisk fysik \\ Box 803, SE-751 08 Uppsala, Sweden \\ ulf@teorfys.uu.se
}

\begin{abstract}
A family of de Sitter vacua introduced in hep-th/0203198 as plausible initial conditions for inflation, are discussed from the point of view of de Sitter holography. The vacua are argued to be physically acceptable and the inflationary picture provides a physical interpretation of a subfamily of de Sitter invariant vacua. Some speculations on the issue of vacuum choice and the connection between the CMBR and holography are also provided.
\end{abstract}

May 2002 


\section{Introduction}

In recent years there has been a significant interest in the use of cosmology as a probe of high energy physics. A well established example is provided by inflation where microscopic quantum fluctuations are magnified to macroscopic scales, and act as seeds for subsequent structure formation in the early universe. For a nice review see [1].

An intriguing possibility is that inflation might provide a window towards physics beyond the Planck scale. In the standard inflationary scenario the fluctuations start out with a linear size much smaller than the Planck scale. Nevertheless it is assumed that no new physics appear, and a natural vacuum for the quantum fluctuations is chosen with this in mind. In several recent works the rationality of this assumption has been questioned, [2-26]. After all, in the real world we know that fundamentally new physics is to be expected at the Planck scale and beyond. The idea is that the fluctuation spectrum might leave an imprint on the CMBR that depends on the details of this transplanckian physics. Several examples exist in the literature of specific modifications of the high energy physics which also give a modified spectrum. In [27] (and more recently in 28]) another point of view was taken with the focus on the choice of vacuum. Initial conditions (for a particular mode) were imposed when the wavelength was comparable to some fundamental length scale in the theory. It was shown that this leads to corrections of order $\frac{H}{\Lambda}$ to the CMBR-spectrum, where $\Lambda$ is the energy scale of new physics, e.g. the Planck scale or the string scale, and $H$ is the Hubble constant during inflation.

The work of [27] only attempted to estimate the natural size of the corrections but had nothing to say about the actual mechanism that determines the vacuum. To do that, one needs knowledge about the high energy physics which goes beyond what is currently available. If one is looking for large scale effects due to quantum gravity it is tempting to consider holography. Holography is well established in the AdS setting [29] 30| 31], while in the case of de Sitter space there are only more or less well founded guesses of what to expect. An incomplete list of references is given by [32-57]. Nevertheless, several interesting and suggestive results have been obtained and the hope is that further work might pin down the principles that governs the physics of the hologram. But even before this goal is achieved, one can embark on the project of completing a holographic dictionary where various aspects of the inflationary theory are expressed in terms of holographic concepts. In [58] [59] [60] it has been shown how, e.g., inflation with a slowly time varying Hubble constant (i.e. the slow roll) can be understood as a renormalization group flow in the hologram.

The main purpose of this note is to translate some of the observations made in [27] into a language appropriate for the holographic studies. In particular it is pointed out that the vacua selected in [27] corresponds to a one-parameter subfamily of the two-parameter family of de Sitter invariant vacua recently discussed in $53 \| 54$. The outline of the paper is as follows. In section two the construction of de Sitter invariant vacua is reviewed. In section three the connection with [27] is pointed out. In section 
four the properties of the vacua relevant for inflation are discussed. Finally, section five contains some conclusions and speculations on possible relations with holography.

\section{A de Sitter family of vacua}

De Sitter space does not have a globally time like Killing vector and the choice of vacuum is therefore a highly non trivial issue [61]. For the study of physics in de Sitter space there are several different coordinate systems available. Below we will consider global coordinates (with spherical spatial sections) that cover the full de Sitter space, and planar coordinates (with flat spatial sections) that only cover half of de Sitter space. In the last section we will make use of yet another type of coordinates, the static coordinates, which are useful when the focus is on observations made by a particular observer.

The planar coordinates are the ones that are relevant for an inflating cosmology, where only half of de Sitter space is physical. The Penrose diagram of de Sitter space

has the shape of a square, and can be viewed as consisting of two parts. An inflating cosmology in the upper right triangle, and a deflating cosmology in the lower left. They are separated by a light like surface corresponding to the Big Bang or the Big Crunch respectively. We will, however, first investigate the choice of vacuum from the point of view global coordinates, closely following the analysis of [53] [54].

De Sitter space in $D$ dimensions can be defined through a hypersurface

$$
-X_{0}^{2}+\sum_{i=1}^{D} X_{i}^{2}=\frac{1}{H^{2}},
$$

in $D+1$ dimensional flat Minkowski space. Global coordinates are obtained by putting

$$
\begin{aligned}
X_{0} & =H^{-1} \sinh H \tau \\
X_{i} & =H^{-1} x_{i} \cosh H \tau,
\end{aligned}
$$

where $\sum_{i=1}^{D} x_{i}^{2}=1$. The corresponding metric, in the case of four dimensional de Sitter space, is given by:

$$
d s^{2}=d \tau^{2}-H^{-2} \cosh ^{2} H \tau d \Omega_{3}^{2} .
$$

Following the usual rules of canonically quantizing a massless scalar field, one finds that

$$
\widehat{\phi}(x)=\sum_{n}\left[\phi_{n}(x) \widehat{a}_{n}+\phi_{n}^{*}(x) \widehat{a}_{n}^{\dagger}\right],
$$

where

$$
\left[\widehat{a}_{n}^{\dagger}, \widehat{a}_{m}\right]=\delta_{n m},
$$

are creation and annihilation operators, and $\phi_{n}(x)$ are modes solving the scalar field equation of motion with $n$ collectively referring to the discrete quantum numbers of 
the spherical harmonics on $S^{3}$. For clarity we will put an hat on all operator valued fields. The vacuum of the theory is defined to obey

$$
\widehat{a}_{n}|\Omega\rangle=0
$$

The vacuum in de Sitter space is not unique, instead, as was first discussed in 662, and later in [63] 64] 65], there is a whole family of possible vacua. More recent discussions, in the context of de Sitter holography, can be found in [53] [54]. Different choices correspond to different mode expansions, like the one in (4), where the different choices are related through Bogolubov transformations. For our study of the various vacua it will be convenient to use the Wightman function defined by

$$
G^{+}\left(x, x^{\prime}\right)=\left\langle\Omega\left|\widehat{\phi}(x) \widehat{\phi}\left(x^{\prime}\right)\right| \Omega\right\rangle=\sum_{n} \phi_{n}(x) \phi_{n}^{*}\left(x^{\prime}\right) .
$$

The Wightman function is a useful building block from which the other Green's functions can be obtained. Through the study of the Wightman function one can learn about the properties of the vacua that one is interested in. A vacuum that will play a special role is the Bunch-Davies vacuum (also known as the Euclidean vacuum) which is obtained by analytical continuation from the Euclidean sphere. It has a Wightman function given by

$$
G_{E}^{+}\left(x, x^{\prime}\right)=\left\langle E\left|\phi(x) \phi\left(x^{\prime}\right)\right| E\right\rangle=\frac{\Gamma\left(h_{+}\right) \Gamma\left(h_{-}\right)}{16 \pi^{2}} F\left(h_{+}, h_{-} ; 2 ; \frac{1+P}{2}\right),
$$

where

$$
h_{ \pm}=\frac{3}{2} \pm \sqrt{\frac{9}{4}-m^{2}}
$$

and $P=P\left(x, x^{\prime}\right)$ is the de Sitter invariant distance. Vacua with this simple space time dependence are called de Sitter invariant and the Bunch-Davies vacuum is therefore an example of such a vacuum.

As discussed in 63 64 one can construct a family of de Sitter invariant vacua by a Bogolubov transformation,

$$
\phi_{n}(x)=A \phi_{n, E}(x)+B \phi_{n, E}^{*}(x),
$$

where

$$
|A|^{2}-|B|^{2}=1
$$

The transformation that we consider treats all modes in the same way (independent of $n$ ) and therefore does not spoil the de Sitter invariance. According to [63] [64] it is possible choose the Euclidean modes such that

$$
\phi_{n, E}^{*}(x)=\phi_{n, E}\left(x_{A}\right)
$$

where $x_{A}$ is the antipodal point. The antipodal map is, in terms of the variables $X_{\mu}$ in (1), obtained by taking $X_{\mu} \rightarrow-X_{\mu}$. In terms of global coordinates one finds that 
$x=(\tau, \Omega)$ is mapped to $x_{A}=\left(-\tau, \Omega_{A}\right) . \Omega_{A}$ is the point opposite to $\Omega$ on $S^{3}$. Note that $P\left(x, x^{\prime}\right)=-P\left(x_{A}, x^{\prime}\right)$. For easy comparison with the case of planar coordinates below, it will be useful to write (10) as

$$
\phi(x)=A \phi_{E}(x)+B \phi_{E}\left(x_{A}\right) .
$$

Using (17) one finds, finally, a Wightman function given by

$$
G^{+}\left(x, x^{\prime}\right)=|A|^{2} G_{E}^{+}\left(x, x^{\prime}\right)+|B|^{2} G_{E}^{+}\left(x^{\prime}, x\right)+A B^{*} G_{E}^{+}\left(x, x_{A}^{\prime}\right)+B A^{*} G_{E}^{+}\left(x_{A}, x^{\prime}\right) .
$$

After this warm up, we will now turn to the case relevant for inflation, i.e. the planar coordinates.

The metric in terms of planar coordinates is given by

$$
d s^{2}=d t^{2}-a(t)^{2} d \mathbf{x}^{2}
$$

where $a(t)=e^{H t}$, is the scale factor. In terms of the conformal time $\eta=-\frac{1}{a H}$ the metric becomes

$$
d s^{2}=\frac{1}{H^{2} \eta^{2}}\left(d \eta^{2}-d \mathbf{x}^{2}\right)
$$

The relation to the hypersurface of (11) is given through

$$
\begin{aligned}
\eta & =-\frac{1}{H^{2}\left(X_{0}+X_{4}\right)} \\
x_{i} & =\frac{X_{i}}{H\left(X_{0}+X_{4}\right)} \quad i=1,2,3,
\end{aligned}
$$

with $X_{0}+X_{4}>0$. As discussed in [54] the Bunch-Davies vacuum has a simple description in planar coordinates. It corresponds to a situation where there are no incoming particles on $\eta \rightarrow-\infty$. As discussed above, the inflationary universe can be viewed as the upper right triangle of the full de Sitter space where $\eta \rightarrow-\infty$ corresponds to the Big Bang, while $\eta \rightarrow 0$ is $\mathcal{I}^{+}$. The lower half of de Sitter space can be covered if we also consider positive $\eta$. In particular, both signs of $\eta$ are allowed in the de Sitter invariant distance, which in planar coordinates becomes

$$
P\left(x, x^{\prime}\right)=\frac{\eta^{2}+\eta^{\prime 2}-\left(\mathbf{x}-\mathbf{x}^{\prime}\right)^{2}}{2 \eta \eta^{\prime}} .
$$

It follows from (??) that the antipodal map in planar coordinates takes $x=(\eta, \mathbf{x})$ into $\bar{x}=(-\eta, \mathbf{x})$. That is, a map between the two halves of de Sitter space. Let us now proceed with the quantization, which is formally very similar to the analysis in global coordinates.

In planar coordinates a massless scalar field can be decomposed as

$$
\widehat{\phi}(x)=\int d^{3} k\left[\phi_{\mathbf{k}}(x) \widehat{a}_{\mathbf{k}}+\phi_{-\mathbf{k}}^{\dagger}(x) \widehat{a}_{\mathbf{k}}^{\dagger}\right] .
$$


We now assume that the modes can be written in terms of the modes defining the Bunch-Davies vacuum through

$$
\phi_{\mathbf{k}}(x)=A \phi_{\mathbf{k}, E}(x)+B \phi_{\mathbf{k}, E}(\bar{x}),
$$

which are the analogue of (13) in planar coordinates. For convenience we write

$$
\phi_{\mathbf{k}, E}(x)=\phi_{k, E}(\eta) e^{i \mathbf{k} \cdot \mathbf{x}}
$$

and demand that a relation analogous to (12) is fulfilled:

$$
\phi_{k, E}(\eta)=\phi_{k, E}^{*}(-\eta) \text {. }
$$

Finally the Wightman function becomes, in complete analogy with the case of global coordinates,

$$
G^{+}\left(x, x^{\prime}\right)=|A|^{2} G_{E}^{+}\left(x, x^{\prime}\right)+|B|^{2} G_{E}^{+}\left(x^{\prime}, x\right)+A B^{*} G_{E}^{+}\left(x, \bar{x}^{\prime}\right)+B A^{*} G_{E}^{+}\left(\bar{x}, x^{\prime}\right) .
$$

We will come back to this expression shortly, to see what it has to tell about the new vacua.

For a massless scalar the above discussion breaks down due to the presence of a zero mode. As pointed out in [64], it is actually impossible to construct de Sitter invariant vacua for a massless field if one restricts the attention to just Fock vacua. This does not, however, mean that de Sitter invariant vacua does not exist. There are two ways to see this. Either one goes beyond the Fock vacuum and includes a piece corresponding to a first quantized particle in one dimension, see [66]. Or, as proposed in [67], one makes sure that all correlation functions that one considers only involve derivatives of the field. The situation is quite similar to the string world sheet theory where the coordinate field $X^{\mu}$ is not a conformal field. There we can nevertheless consider insertions of operators of the form $\partial X^{\mu}$, or, with a little more thought, operators of the form $e^{i p_{\mu} X^{\mu}}$.

In the present paper we will be working in momentum space where the subtleties of the massless case are irrelevant as long as we stay away from vanishing momentum. Hence the problems of the zero mode do not appear.

\section{Interpreting the vacua}

In this section we will give an interpretation of a one parameter subfamily of the two parameter family of vacua described in the previous section. To be explicit, we have, for a massless field in planar coordinates,

$$
\phi_{k, E}(\eta)=-\eta H \frac{1}{\sqrt{2 k}} e^{-i k \eta}\left(1-\frac{i}{k \eta}\right),
$$


and new modes given by

$$
\phi_{k}(\eta)=\frac{-\eta H}{\sqrt{2 k}}\left(A e^{-i k \eta}\left(1-\frac{i}{k \eta}\right)+B e^{i k \eta}\left(1+\frac{i}{k \eta}\right)\right) .
$$

We also need the conjugate momentum which is given by

$$
\pi_{k}(\eta)=\phi_{k}^{\prime}(\eta)=\frac{i k \eta H}{\sqrt{2 k}}\left(A e^{-i k \eta}-B e^{i k \eta}\right) .
$$

As reviewed in the previous section, there is (up to an overall phase) a two parameter family of de Sitter invariant vacua. We will now focus on a particular one parameter subfamily such that:

$$
\begin{aligned}
A & =e^{i(\theta-\beta)} \frac{2 \beta-i}{2 \beta} \\
B & =-e^{i(\theta+\beta)} \frac{i}{2 \beta}
\end{aligned}
$$

where $\beta$ and $\theta$ (the overall phase) are both real. Note that definitions are such that $|A|^{2}-|B|^{2}=1$. The crucial properties of these vacua, parametrized by $\beta$, is that they imply the existence of a particular moment in time when there is a very simple relation between $\phi_{k}$ and $\pi_{k}$. One easily verifies that

$$
\begin{aligned}
\phi_{k}\left(\eta_{k}\right) & =\frac{-\eta_{k} H}{\sqrt{2 k}} e^{i \theta} \\
\pi_{k}\left(\eta_{k}\right) & =-i k \phi_{k}\left(\eta_{k}\right),
\end{aligned}
$$

where

$$
\eta_{k}=-\frac{\beta}{k}
$$

Note that the conformal time $\eta_{k}$ depends on $k$. Since $\eta=-\frac{1}{a H}$ we find that

$$
p=\frac{k}{a}=\beta H,
$$

where $p$ is the physical momentum. If we then identify $\beta=\frac{\Lambda}{H}$, we find that the physical momentum, corresponding to the mode $k$, is equal to $\Lambda$ at conformal time $\eta_{k}$. The vacua defined by (28) therefore have the property that a mode $k$ is created in a state of the form (29) at the time $\eta_{k}$ when its physical size is given by the universal scale $\Lambda$.

There are several ways to characterize a state of the form (29). As pointed out in [27], following [69], one can note that it corresponds to a state of minimum uncertainty. By construction it is also what might be called a local Minkowski vacuum defined through

$$
\widehat{a}_{\mathbf{k}}|\Omega\rangle=0
$$


where $\widehat{a}_{\mathbf{k}}$ is the annihilation operator appearing in (20).

The analysis in [27] proceeded further by calculating the perturbation spectrum that would result from these initial conditions. In the case of $\frac{\Lambda}{H} \gg 1$ it was shown that the fluctuation spectrum for this new vacuum becomes

$$
P_{\phi}=\left\langle\left|\phi_{k}(\eta)\right|^{2}\right\rangle=\left(\frac{H}{2 \pi}\right)^{2}\left(1-\frac{H}{\Lambda} \sin \left(\frac{2 \Lambda}{H}\right)\right),
$$

which shows that the natural size of a transplanckian correction is given by $\frac{H}{\Lambda}$ in agreement with the claims in [12] [15]. In a model of inflation where $H$ is slowly changing, with a spectrum which is not exactly scale invariant, the correction term will be very sensitive to $k$ through the dependence of $H$ on $k$. That is, there will be a modulation of $P_{\phi}$. This could be a rather general phenomena in models where the initial conditions are set at a particular scale. The modulation that was found is precisely of the same form as in the numerical work of [15] which considered a specific example of slow roll. In further work by the same authors, [28], the analytical approach has been extended to the case of a varying Hubble constant.

In conclusion we have found a very simple interpretation of a subfamily of de Sitter invariant vacua. It would be interesting to see whether the same holds true in general.

\section{Some properties of the de Sitter family of vacua}

In the previous section it was shown that the vacuum discussed in [27], which is of interest from the point of view of transplanckian effects, is among the family of de Sitter invariant vacua discussed in [53] [54]. This is a simple consequence of the way the initial conditions are chosen with all modes treated equivalently.

The construction of the vacuum, through the use of initial conditions at a specific scale, provides a physical way of understanding the nature of the de Sitter invariant vacua. From the opposite point of view, the analysis of section 2 can give information about the properties of these vacua and tell whether they make physical sense or not. There are three points to have in mind in this context.

1. The commutator is independent of the choice of vacuum and the same is true for the retarded and advanced Greens functions. As a consequence the classical evolution remains the same. However, if we consider the Wightman function, or the Feynman propagator, there will be new singularities on the light cone of the image source at $x_{A}$. It is therefore tempting to exclude these new vacua as recently discussed in, e.g., 688. But it is easy to see that the singularities only appear for points outside of their respective de Sitter horizons. Local measurements within the horizon will therefore not encounter any peculiar singularities. The main effect is simply that effectively new initial conditions on $\eta \rightarrow-\infty$ have been imposed using an image charge formally positioned on the wrong half of de Sitter. 
2. The various de Sitter invariant vacua are physically different. An example of such a difference is that only the Bunch-Davies vacuum is thermal. This was discussed in [53] where it was shown that a freely falling detector will only come into thermal equilibrium in the Bunch-Davies vacuum. It is also quite obvious from the explicit expressions for the Wightman function that it does not have the analyticity properties characteristic for a thermal system.

3. At high energies (short distances) the de Sitter invariant vacua (except the Bunch-Davies) are in general different from ordinary Minkowski space (even if there are no new singularities). This is expected and actually the whole point with the reasoning at the end of the previous section. It simply means that we encounter transplanckian effects when we probe short distances. The standard argument would have been that the Greens function should approach the usual Minkowski expression when the distance is small enough compared to the Hubble scale. However, for small distances we encounter new physics and there is no regime where the Greens function will take the Minkowski form.

To conclude, there is no reason of principle to limit one self to the Bunch-Davies vacuum, at least if one is interested in an inflating cosmology where only half of de Sitter space is relevant.

\section{Conclusions and speculations on holography}

In this paper we have observed that the vacua discussed in [27] are among the family of de Sitter invariant vacua explored in [53][54]. We have also pointed out that there is no obvious reason to exclude these vacua from a physical point of view, and a better knowledge of high energy physics is needed in order to make the correct choice. It would be interesting if the question of how the vacuum is determined could be made in a holographic setting. In this respect one should note that the holographic counterpart of the CMBR-fluctuation spectrum is given by two point correlator in the holographic theory.

In the by now standard AdS/CFT holographic correspondence, the hologram is situated at the boundary of AdS. The boundary has a Lorentzian signature and the theory living there is in many cases a fairly standard CFT. A similar relation is supposed to hold also in the de Sitter case, where the boundary is either in the infinite future, $\mathcal{I}^{+}$, or in the infinite past, $\mathcal{I}^{-}$. A crucial difference from the AdS is that the boundary has an Euclidean signature. Since we are interested in applying the construction to inflation we will focus on $\mathcal{I}^{+}$. As we approach $\mathcal{I}^{+}$, as $\eta \rightarrow$ 0 , it therefore becomes inappropriate to divide the field according to positive and negative frequency, see [57]. Rather we should distinguish between the two possible asymptotic behaviors with $\phi \sim \eta^{h_{ \pm}}$, implying that there are actually two operators in the boundary conjugate to a given bulk field. We denote these operators by $\mathcal{O}_{\mathbf{k}}^{+}$ 
and $\mathcal{O}_{\mathrm{k}}^{-}$in momentum space. For the two point functions one therefore finds that

$$
\left\langle\widehat{\phi}_{\mathbf{k}}(\eta) \widehat{\phi}_{-\mathbf{k}}\left(\eta^{\prime}\right)\right\rangle=\eta^{h_{-}} \eta^{h_{-}}\left\langle\mathcal{O}_{\mathbf{k}}^{-} \mathcal{O}_{-\mathbf{k}}^{-}\right\rangle+\eta^{h_{+}} \eta^{h_{+}}\left\langle\mathcal{O}_{\mathbf{k}}^{+} \mathcal{O}_{-\mathbf{k}}^{+}\right\rangle \sim\left\langle\mathcal{O}_{\mathbf{k}}^{-} \mathcal{O}_{-\mathbf{k}}^{-}\right\rangle,
$$

with $h_{+}=3$ and $h_{-}=0$ for a massless bulk field, as we approach the boundary. It follows, therefore, that the fluctuation spectrum relevant for the CMBR is, from a holographic point of view, directly given by the correlator $\left\langle\mathcal{O}_{\mathbf{k}}^{-} \mathcal{O}_{-\mathbf{k}}^{-}\right\rangle$. The main observation is that the holographic screen on $\mathcal{I}^{+}$corresponds to the frozen modes that have expanded outside of the inflationary horizon. If inflation never stopped, they would not be associated with anything measurable. In 33. such quantities on $\mathcal{I}^{+}$ were referred to as meta-observables. However, in the real universe, the modes re-enter through the horizon after the end of inflation and act like seeds for the fluctuation spectrum of the CMBR. The study of the CMBR therefore corresponds to a study of the theory on $\mathcal{I}^{+}$, and the meta-observables actually turns into real observables accessible to measurements. This picture, however, breaks down for small scales on $\mathcal{I}^{+}$, corresponding to late times, when inflation turns off.

The sensitivity to the initial conditions of inflation explored in [27] has a precise counterpart in the dependence on the vacuum of the holographic correlation functions as discussed in [53] [54, and the question arises what holography might have to say about the choice of vacuum. The family of de Sitter invariant vacua all correspond to scale invariant vacua in the theory on $\mathcal{I}^{+}$. Thinking in terms of planar coordinates, identifying the comoving momentum $\mathbf{k}$ with the Euclidean momentum in the hologram, the time evolution (in conformal time) corresponds to an inverted renormalization group flow from large to small scales. As the flow proceeds, new modes are integrated in requiring initial conditions. Clearly this happens, for some particular $\mathbf{k}$, when the corresponding wavelength becomes of the same order as the cutoff.

To proceed further we need to know a bit more about the de Sitter holography. A useful coordinate system that brings the focus on what a particular observer will experience, is the static coordinate system. (A discussion of the relation between planar and static coordinates from the point of view of dS/CFT can be found in 48). The metric in static coordinates is given by

$$
d s^{2}=-\left(1-H^{2} r^{2}\right) d t^{2}+\frac{d r^{2}}{1-H^{2} r^{2}}+r^{2} d \Omega_{d-2}^{2}
$$

where $r<1 / H$ corresponds to the causal diamond [70], i.e. the region of space time which the observer can influence or get influenced by. If we use planar coordinates such that

$$
d s^{2}=\frac{1}{H^{2} \eta^{2}}\left(-d \eta^{2}+d \rho^{2}+\rho^{2} d \Omega_{d-2}^{2}\right)
$$

the coordinate transformation that takes us between the planar and static coordinates is given by

$$
\begin{aligned}
\rho & =\rho(t, r)=-H r \eta \\
\eta(t, r) & =-\frac{e^{-H t}}{H \sqrt{H^{2} r^{2}-1}} .
\end{aligned}
$$


In planar coordinates it follows from the metric (16) that a source at $\eta$ will give rise to a blob of size $\Delta x=|\eta|$ in the boundary at $\mathcal{I}^{+}$. It is therefore natural to associate a cutoff of order $|\eta|$ to a spacelike section at conformal time $\eta$ (the same all over $\mathcal{I}^{+}$). If we now make a coordinate transformation on the boundary, mapping the plane into a cylinder, several things will happen. All circles, independent of their radius $\rho$ in planar coordinates, will be mapped onto the circumference of a cylinder with a radius independent of the direction $t$ along the cylinder. If we want a cutoff that is the same all over the cylinder (rather than all over the plane) we need to change the original cutoff in order to keep $\rho / \eta$ constant. This will imply a constant $r$ through (37), with a cutoff on the cylinder that is proportional to $1 / r$. From (38) we can read off what a particular cutoff corresponds to in terms of $\eta$. We find that late times (large $t$ ) along the cylinder corresponds to small $\eta$.

The cylinder corresponds to a surface of radius $r>1 / H$ outside the cosmological horizon, but if we want the theory to precisely describe the degrees of freedom on the horizon we need to take $r=1 / H$. It follows from, e.g., (37) that the cutoff now is comparable to the radius of the cylinder, and there will be only one lattice point encoding all the degrees of freedom of the whole horizon. Luckily, due to the large central charge the theory has many degrees of freedom living at each of these lattice points. This analysis is in line with the analysis in 71 .

The above discussion makes it clear that the horizon has, at $\eta$, a size $\Delta x=|\eta|$ implying that the resolution of the hologram is such that there is only one lattice point per horizon volume. The vacua that we have been discussing implies that the initial conditions are imposed, at time $\eta$, on scales given by

$$
\Delta x=\frac{H}{\Lambda}|\eta|,
$$

which is considerably smaller than the resolution of the holographic theory. It follows that the choice of vacuum is encoded in the, say, $N$ degrees of freedom at the lattice point. If, furthermore, $\Lambda$ is related to the Planck scale (which, for fixed $H$, also determines the number of degrees of freedom in the holographic theory), it follows that any transplanckian effects must be associated with $1 / N$ effects in the choice of the vacuum.

Clearly it would be very interesting if one could find a way to select a natural vacuum from the point of view of the hologram. A specific problem in this context is to characterize the minimum uncertainty vacua, discussed in section 3 , from a holographic point of view. We hope to return to these and related questions in the near future.

\section{Acknowledgments}

I would like to thank Hector Rubinstein and Alexei Abrikosov for comments and

discussions. The author is a Royal Swedish Academy of Sciences Research Fellow 
supported by a grant from the Knut and Alice Wallenberg Foundation. The work was also supported by the Swedish Research Council (VR).

\section{References}

[1] A. R. Liddle and D. H. Lyth, "Cosmological inflation and large-scale structure", Cambridge University Press 2000.

[2] R. H. Brandenberger, "Inflationary cosmology: Progress and problems," arXiv:hep-ph/9910410.

[3] J. Martin and R. H. Brandenberger, "The trans-Planckian problem of inflationary cosmology," Phys. Rev. D 63, 123501 (2001) arXiv:hep-th/0005209.

[4] J. C. Niemeyer, "Inflation with a high frequency cutoff," Phys. Rev. D 63, 123502 (2001) arXiv:astro-ph/0005533.

[5] R. H. Brandenberger and J. Martin, "The robustness of inflation to changes in super-Planck-scale physics," Mod. Phys. Lett. A 16, 999 (2001) arXiv:astroph/0005432].

[6] A. Kempf, "Mode generating mechanism in inflation with cutoff," Phys. Rev. D 63, 083514 (2001) arXiv:astro-ph/0009209.

[7] C. S. Chu, B. R. Greene and G. Shiu, "Remarks on inflation and noncommutative geometry," Mod. Phys. Lett. A 16, 2231 (2001) arXiv:hep-th/0011241.

[8] J. Martin and R. H. Brandenberger, "A cosmological window on trans-Planckian physics," arXiv:astro-ph/0012031.

[9] L. Mersini, M. Bastero-Gil and P. Kanti, "Relic dark energy from trans-Planckian regime," Phys. Rev. D 64, 043508 (2001) arXiv:hep-ph/0101210.

[10] J. C. Niemeyer and R. Parentani, "Trans-Planckian dispersion and scaleinvariance of inflationary perturbations," Phys. Rev. D 64, 101301 (2001) arXiv:astro-ph/0101451.

[11] A. Kempf and J. C. Niemeyer, "Perturbation spectrum in inflation with cutoff," Phys. Rev. D 64, 103501 (2001) arXiv:astro-ph/0103225.

[12] R. Easther, B. R. Greene, W. H. Kinney and G. Shiu, "Inflation as a probe of short distance physics," Phys. Rev. D 64, 103502 (2001) arXiv:hep-th/0104102.

[13] M. Bastero-Gil and L. Mersini, "SN1A data and CMB of Modified Curvature at Short and Large Distances," Phys. Rev. D 65 (2002) 023502 arXiv:astroph/0107256]. 
[14] L. Hui and W. H. Kinney, "Short distance physics and the consistency relation for scalar and tensor fluctuations in the inflationary universe," arXiv:astroph/0109107.

[15] R. Easther, B. R. Greene, W. H. Kinney and G. Shiu, "Imprints of short distance physics on inflationary cosmology," arXiv:hep-th/0110226.

[16] M. Bastero-Gil, P. H. Frampton and L. Mersini, "Modified dispersion relations from closed strings in toroidal cosmology," arXiv:hep-th/0110167.

[17] R. H. Brandenberger, S. E. Joras and J. Martin, "Trans-Planckian physics and the spectrum of fluctuations in a bouncing universe," arXiv:hep-th/0112122.

[18] J. Martin and R. H. Brandenberger, "The Corley-Jacobson dispersion relation and trans-Planckian inflation," arXiv:hep-th/0201189.

[19] J. C. Niemeyer, "Cosmological consequences of short distance physics," arXiv:astro-ph/0201511.

[20] F. Lizzi, G. Mangano, G. Miele and M. Peloso, arXiv:hep-th/0203099.

[21] G. Shiu and I. Wasserman, "On the signature of short distance scale in the cosmic microwave background," arXiv:hep-th/0203113.

[22] R. Brandenberger and P. M. Ho, "Noncommutative spacetime, stringy spacetime uncertainty principle, and density fluctuations," arXiv:hep-th/0203119.

[23] S. Shankaranarayanan, "Is there an imprint of Planck scale physics on inflationary cosmology?," arXiv:gr-qc/0203060.

[24] N. Kaloper, M. Kleban, A. E. Lawrence and S. Shenker, "Signatures of short distance physics in the cosmic microwave background," arXiv:hep-th/0201158.

[25] R. H. Brandenberger and J. Martin, "On signatures of short distance physics in the cosmic microwave background," arXiv:hep-th/0202142.

[26] S. F. Hassan and M. S. Sloth, "Trans-Planckian effects in inflationary cosmology and the modified uncertainty principle," arXiv:hep-th/0204110.

[27] U. H. Danielsson, "A note on inflation and transplanckian physics," arXiv:hepth/0203198. Accepted for publication by Physical Review D.

[28] R. Easther, B. R. Greene, W. H. Kinney and G. Shiu, "A generic estimate of trans-Planckian modifications to the primordial power spectrum in inflation," arXiv:hep-th/0204129.

[29] J. Maldacena, "The large $N$ limit of superconformal field theories and supergravity," Adv. Theor. Math. Phys. 2, 231 (1998) [Int. J. Theor. Phys. 38, 1113 (1998)] arXiv:hep-th/9711200. 
[30] S. S. Gubser, I. R. Klebanov and A. M. Polyakov, "Gauge theory correlators from non-critical string theory," Phys. Lett. B 428, 105 (1998) arXiv:hepth/9802109].

[31] E. Witten, "Anti-de Sitter space and holography," Adv. Theor. Math. Phys. 2, 253 (1998) arXiv:hep-th/9802150.

[32] C. M. Hull, "Timelike T-duality, de Sitter space, large N gauge theories and topological field theory," JHEP 9807 (1998) 021 arXiv:hep-th/9806146.

[33] E. Witten, "Quantum gravity in de Sitter space," arXiv:hep-th/0106109.

[34] A. Strominger, "The ds/CFT correspondence," arXiv:hep-th/0106113.

[35] V. Balasubramanian, P. Horava and D. Minic, "Deconstructing de Sitter," JHEP 0105 (2001) 043 arXiv:hep-th/0103171.

[36] M. Li, "Matrix model for de Sitter," JHEP 0204, 005 (2002) arXiv:hepth/0106184.

[37] S. Nojiri and S. D. Odintsov, "Conformal anomaly from dS/CFT correspondence," Phys. Lett. B 519 (2001) 145 arXiv:hep-th/0106191.

[38] D. Klemm, "Some aspects of the de Sitter/CFT correspondence," arXiv:hepth/0106247.

[39] S. Nojiri and S. D. Odintsov, "Quantum cosmology, inflationary brane-world creation and dS/CFT correspondence," JHEP 0112, 033 (2001) arXiv:hepth/0107134.

[40] T. Shiromizu, D. Ida and T. Torii, "Gravitational energy, dS/CFT correspondence and cosmic no-hair," JHEP 0111, 010 (2001) arXiv:hep-th/0109057.

[41] C. M. Hull, "de Sitter space in supergravity and M theory," arXiv:hepth/0109213.

[42] M. Spradlin, A. Strominger and A. Volovich, "Les Houches lectures on de Sitter space," arXiv:hep-th/0110007.

[43] B. McInnes, "Exploring the similarities of the dS/CFT and AdS/CFT correspondences," Nucl. Phys. B 627, 311 (2002) arXiv:hep-th/0110062.

[44] V. Balasubramanian, J. de Boer and D. Minic, "Mass, entropy and holography in asymptotically de Sitter spaces," arXiv:hep-th/0110108.

[45] Y. S. Myung, "Entropy of the three dimensional Schwarzschild-de Sitter black hole," arXiv:hep-th/0110123. 
[46] B. G. Carneiro da Cunha, "Three-dimensional de Sitter gravity and the correspondence," Phys. Rev. D 65, 104025 (2002) arXiv:hep-th/0110169.

[47] R. G. Cai, Y. S. Myung and Y. Z. Zhang, "Check of the Mass Bound Conjecture in de Sitter Space," arXiv:hep-th/0110234.

[48] U. H. Danielsson, "A black hole hologram in de Sitter space," JHEP 0203 (2002) 020 arXiv:hep-th/0110265.

[49] S. Ogushi, "Holographic entropy on the brane in de Sitter Schwarzschild space," Mod. Phys. Lett. A 17, 51 (2002) arXiv:hep-th/0111008.

[50] A. C. Petkou and G. Siopsis, "dS/CFT correspondence on a brane," JHEP 0202, 045 (2002) arXiv:hep-th/0111085.

[51] R. G. Cai, "Cardy-Verlinde formula and asymptotically de Sitter spaces," Phys. Lett. B 525 (2002) 331 arXiv:hep-th/0111093.

[52] A. M. Ghezelbash and R. B. Mann, "Action, mass and entropy of Schwarzschildde Sitter black holes and the de Sitter / CFT correspondence," JHEP 0201, 005 (2002) arXiv:hep-th/0111217.

[53] R. Bousso, A. Maloney and A. Strominger, "Conformal vacua and entropy in de Sitter space," arXiv:hep-th/0112218.

[54] M. Spradlin and A. Volovich, "Vacuum states and the S-matrix in dS/CFT," arXiv:hep-th/0112223.

[55] R. G. Cai, "Cardy-Verlinde formula and thermodynamics of black holes in de Sitter spaces," Nucl. Phys. B 628 (2002) 375 arXiv:hep-th/0112253.

[56] A. M. Ghezelbash, D. Ida, R. B. Mann and T. Shiromizu, "Slicing and brane dependence of the (A)dS/CFT correspondence," arXiv:hep-th/0201004.

[57] S. R. Das, "Thermality in de Sitter and holography," arXiv:hep-th/0202008.

[58] A. Strominger, "Inflation and the dS/CFT correspondence," JHEP 0111, 049 (2001) arXiv:hep-th/0110087.

[59] F. Larsen, J. P. van der Schaar and R. G. Leigh, "de Sitter holography and the cosmic microwave background," JHEP 0204 (2002) 047 [arXiv:hep-th/0202127.

[60] E. Halyo, "Holographic inflation," arXiv:hep-th/0203235.

[61] G. W. Gibbons and S. W. Hawking, "Cosmological Event Horizons, Thermodynamics, And Particle Creation," Phys. Rev. D 15, 2738 (1977).

[62] N. A. Chernikov and E. A. Tagirov, "Quantum theory of scalar field in de Sitter space-time," Ann. Inst. Henri Poincaré, vol. IX, nr 2, (1968) 109. 
[63] E. Mottola, "Particle Creation In De Sitter Space," Phys. Rev. D 31 (1985) 754.

[64] B. Allen, "Vacuum States In De Sitter Space," Phys. Rev. D 32 (1985) 3136.

[65] R. Floreanini, C. T. Hill and R. Jackiw, "Functional Representation For The Isometries Of De Sitter Space," Annals Phys. 175 (1987) 345.

[66] K. Kirsten and J. Garriga, "Massless Minimally Coupled Fields In De Sitter Space: O(4) Symmetric States Versus De Sitter Invariant Vacuum," Phys. Rev. D 48 (1993) 567 arXiv:gr-qc/9305013].

[67] A. J. Tolley and N. Turok, "Quantization of the massless minimally coupled scalar field and the dS/CFT correspondence," arXiv:hep-th/0108119.

[68] A. J. Tolley and N. Turok, "Quantum fields in a big crunch / big bang spacetime," arXiv:hep-th/0204091.

[69] D. Polarski and A. A. Starobinsky, "Semiclassicality and decoherence of cosmological perturbations," Class. Quant. Grav. 13, 377 (1996) arXiv:gr-qc/9504030.

[70] R. Bousso, "Positive vacuum energy and the N-bound," JHEP 0011, 038 (2000) arXiv:hep-th/0010252; R. Bousso, "Bekenstein bounds in de Sitter and flat space," JHEP 0104, 035 (2001) arXiv:hep-th/0012052.

[71] D. Kabat and G. Lifschytz, "De Sitter entropy from conformal field theory," JHEP 0204 (2002) 019 arXiv:hep-th/0203083. 